\title{
ANÁlisis DE ESTRATEGIAS PARA CONFORT TÉRMICO Y LUMÍNICO DE EDIFICIOS EN DIFERENTES CLIMAS DE LA ZONA CENTRAL DEL CHILE
}

WALDo BUSTAMANTE - FELIPE ENCINAS BUSTAMANTE - ROBERTo OtÁRola - ALAN PINO

Waldo Bustamante / Ingeniero Civil Mecánico, Universidad de Chile; Magíster en Desarrollo Urbano, Pontificia Universidad Católica de Chile; Doctor en Ciencias Aplicadas, Université Catholique de Louvain. / Profesor, Escuela de Arquitectura, UC.

Felipe Encinas Bustamante / Arquitecto, Pontificia Universidad Católica de Chile, 2002; Máster en Ciencias, Universidad de Nottingham, 2004; Doctor en Arquitectura Universidad Católica de Lovaina, 2012. Su línea de investigación está asociada al comportamiento térmico y desempeño energético de edificaciones. / Actualmente es profesor de la Escuela de Arquitectura de la Pontificia Universidad Católica de Chile.

Roberto Otárola / Ingeniero Comercial, Universidad de Chile; Candidato a Magister en Ingeniería de la Energía, Pontificia Universidad Católica de Chile.

Alan Pino / Licenciado en Ciencias de la Inge-niería, Pontificia Universidad Católica de Chile; Candidato a Ingeniero Civil Mecánico y Magister en Ciencias de la Ingeniería, Pontificia Universidad Católica de Chile.

Este ejercicio se avoca a la evaluación de tres edificios de oficina, estadísticamente representativos, de la zona central Chile. Dos de ellos se ubican en clima mediterráneo ${ }^{1}$ (ECM01 y ECM02) y el tercero en clima costero $^{2}$ (ECC01). En conjunto abarcan vastas zonas de dicha región (FIGS.01 A 04). En clima mediterráneo, uno de los edificios (ECM01) es de fachada completamente transparente (FIG.05) y, el otro (ECM02), de fachada mixta (FIG.15). En clima costero también se estudió un edificio completamente vidriado (ECC01) (FIG.11).

Para cada caso se analizó el desempeño térmico y lumínico, tanto en su situación original como con mejoramientos aplicados, para conocer la variación en su desempeño según cada tipo de edificio. El desempeño térmico se evaluó en régimen dinámico con el software de simulación TAS. Se consideran las demandas de energía de calefacción y de refrigeración y se observa también, en algunos casos, la variación de temperatura interior, para ciertos recintos en días característicos. El desempeño lumínico se analizó en distintos períodos del año con los software Ecotect y Radiance. Se aplicó además ventilación nocturna como estrategia de enfriamiento. TRANSPARENCIA TOTAL / En los casos ECM01 y ECC01, se observó que la demanda de energía de refrigeración fue significativa- mente más alta que la de calefacción (FIGS.08 y 13). En ECM01, la variación de temperatura interior en un día típico de verano mostró severos problemas de sobrecalentamiento (FIG.06). Este mismo fenómeno se observó en algunos días despejados de invierno (FIG.08), lo que indica la necesidad, aun en este período del año, de utilizar sistemas de refrigeración. Se supuso la aplicación de protección solar en ambos edificios a través de la incorporación de celosías en el exterior (FIGS.08 y 13) para disminuir la demanda de refrigeración, la cual siguió siendo alta en comparación al edificio de envolvente mixta (FIG.18).

A los problemas de sobrecalentamiento se suma el deslumbramiento que provoca la falta de confort lumínico, según se desprende del bajo índice de iluminancia natural útil (UDI, Usefull Daylight Illuminance). Este fenómeno es mayor en el caso ECM01 con DVH claro, que con DVH selectivo (FIGs.09 Y 10). En el caso ECC01 también se observaron severos problemas de deslumbramiento, aun cuando se trata de un clima con mayores niveles de nubosidad y de una fachada con vidrio selectivo (FIG.14).

TRANSPARENCIA PARCIAL / El caso ECM02 mostró una demanda de calefacción menor a la de refrigeración. Esto confirma que en la zona mediterránea de Chile central, las solicitaciones climáticas más críticas en edificios de ofi-

1 Clima templado, con invierno de cuatro a cinco meses, verano y estaciones intermedias con oscilación térmica moderada entre día y noche, y radiación solar intensa; nubosidad baja en verano.

2 Clima templado con influencia marítima, inviernos de cuatro a cinco meses; oscilación térmica baja; nubosidad matinal en verano que disipa al mediodía.
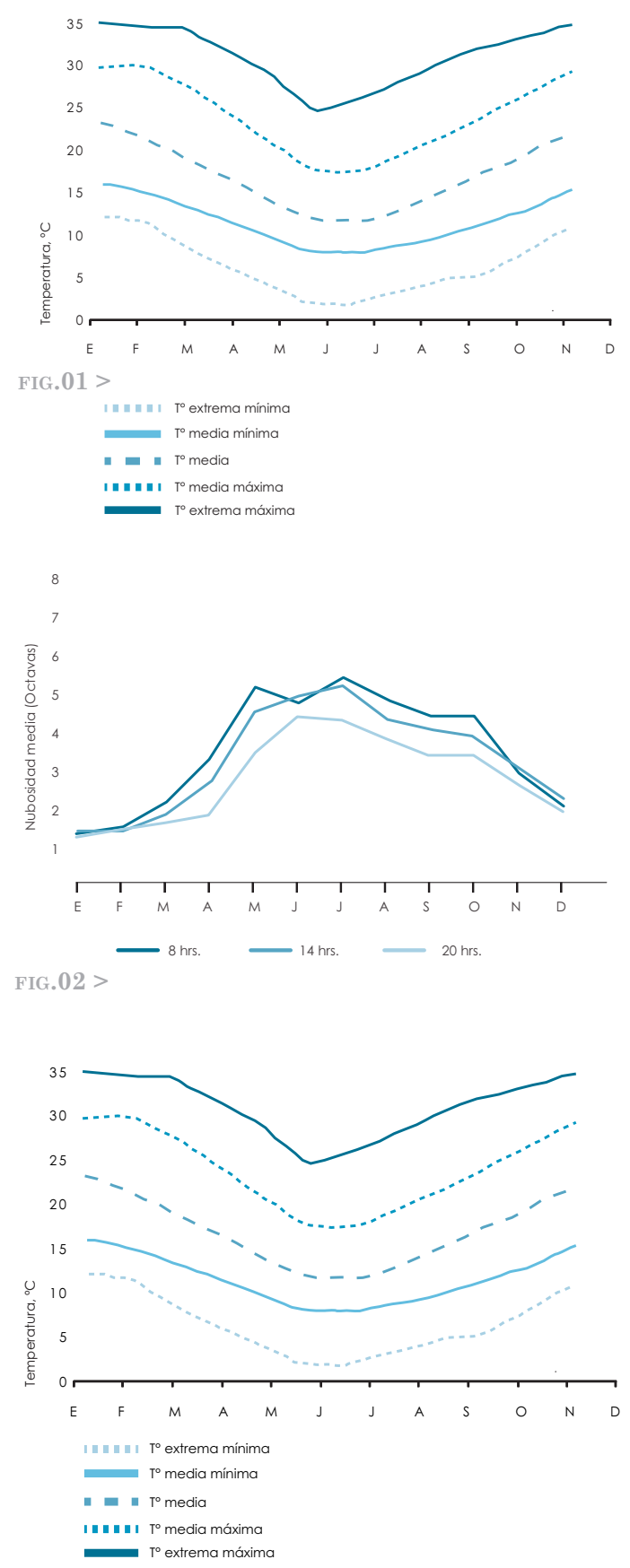

FIG, $03>$

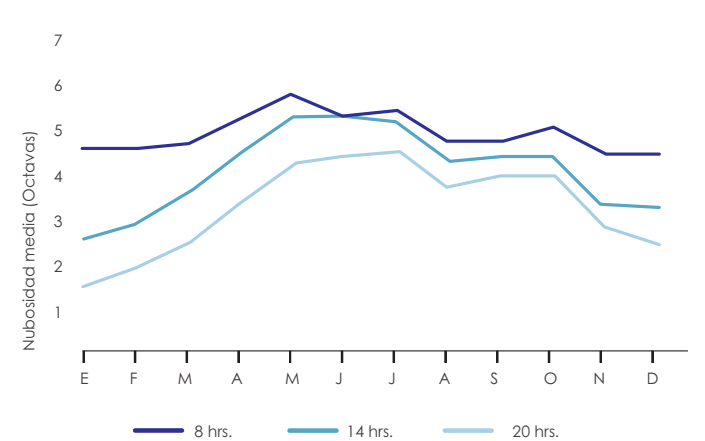

FIG.04 > 
FIG.01 Variación mensual de temperatura media, clima mediterráneo. Fuente: archivo Waldo Bustamante.

FIG.02 Variación mensual de nubosidad media, clima medi terráneo. Fuente: archivo Waldo Bustamante. tero. Fuente: archivo Waldo Bustamante.

FIG.04 Variación mensual de nubosidad media, clima costero. Fuente: archivo Waldo Bustamante.
FIG.03 Variación mensual de temperatura media, clima cos-

HG 5 a 10 ECM01. Edificio en clima mediterráneo 1. Fuente: elaboración propia.

Especificaciones técnicas:

Muro cortina: doble vidriado hermético (DVH) de tipo selectivo; Cristal Sun Energy Gvb de 6 mm, 12 mm de cámara de aire y vidrio claro de $4 \mathrm{~mm}$. Transmitancia solar (TS) = 0,4 . Factor de transmisión de luz $(\mathrm{TL})=0,5$. Transmitancia térmica $(\mathrm{U})=2,8 \mathrm{~W} / \mathrm{m} 2^{\circ} \mathrm{C}$.

Piso sobre subterráneo: hormigón armado (HA) de $150 \mathrm{~mm}$ con recubrimiento de baldosa cerámica. Transmitancia tér $\operatorname{mica}(\mathrm{U})=2,31 \mathrm{~W} / \mathrm{m} 2{ }^{\circ} \mathrm{C}$.

Cubierta: hormigón armado (HA) de $150 \mathrm{~mm}$ y poliestireno expandido (EPS), de densidad $10 \mathrm{~kg} / \mathrm{m} 3$ y $80 \mathrm{~mm}$ de espesor. Transmitancia térmica $(\mathrm{U})=0,48 \mathrm{~W} / \mathrm{m} 2{ }^{\circ} \mathrm{C}$

FIG.05 Esquema y planta.

FIG.06 Variación de temperatura interior en recintos Este (of. A) y Oeste (of. B) en piso 10, en verano.
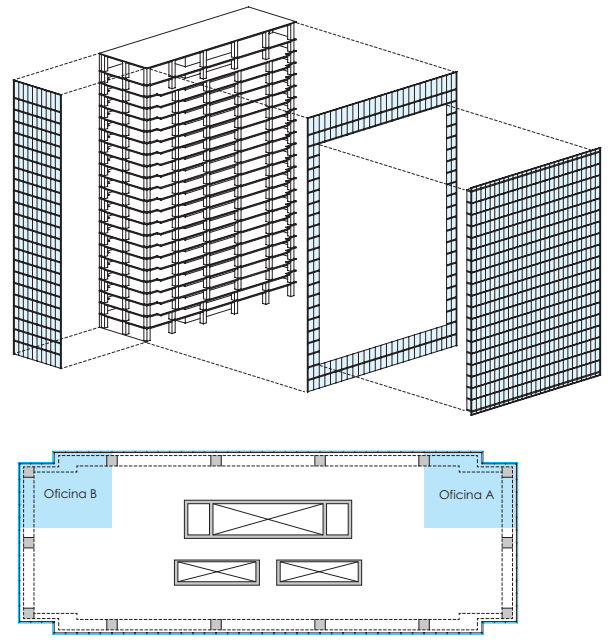

FIG.05 >

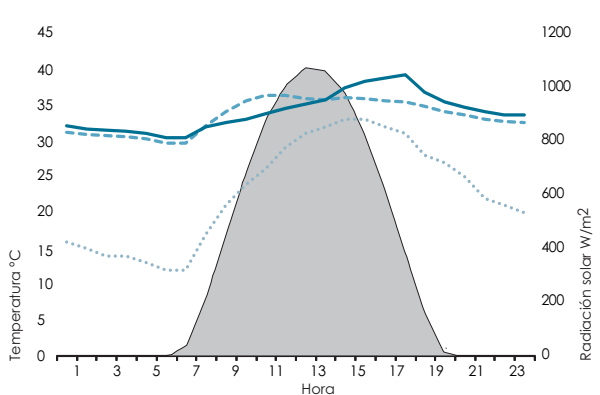

\section{emperatura extern}

Of. A (Oeste
Of B (Este)

Radiación solar globa

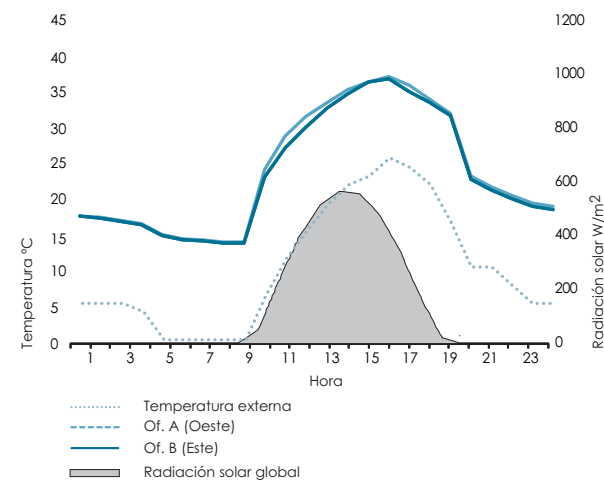

FIG.07 >
Fic.07 Variación de temperatura interior en recintos Este (of. A) y Oeste (of. B) en piso 10, en invierno.

FIG.08 Demandas de energía de acondicionamiento térmico, según diferentes escenarios:

Escenario 1: proyecto original.

Escenario 2: proyecto original con protección solar. Alero en fachada norte y celosías opacas verticales, a 0,5 m del muro, en fachadas oriente y poniente.

Escenario 3: escenario 2 con ventilación nocturna -renovación de aire de ocho volúmenes por hora, entre 22 y 07 hrs. del día siguiente-, entre octubre y marzo.

FIG.09 Iluminancia natural útil (UDI) para rango entre 100 y 2.000 lux en un recinto interior del edificio. Muro cortina con vidrio DVH claro. Líneas de contorno de UDI: 100 a 2.000 lux cada $10 \%$

FIG.10 Iluminancia natural útil (UDI) para rango entre 100 y 2000 lux en un recinto interior del edificio. Muro cort na con doble vidriado hermético (DVH) selectivo. Líneas de contorno de UDI: 100 a 2.000 lux cada $10 \%$.

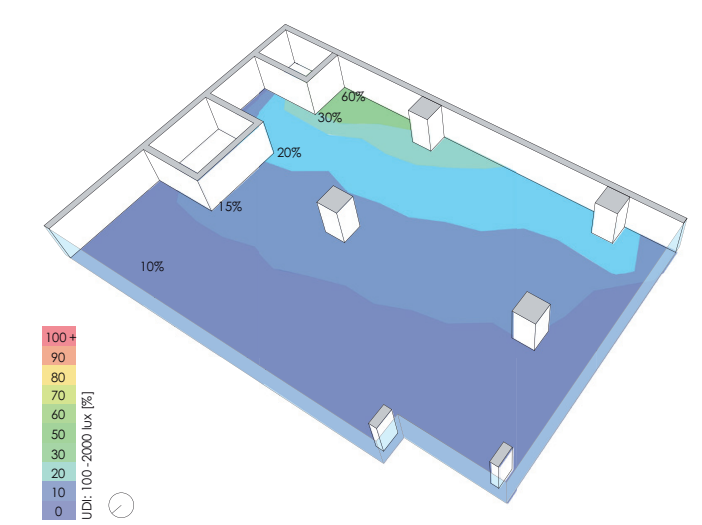

FIG.09>

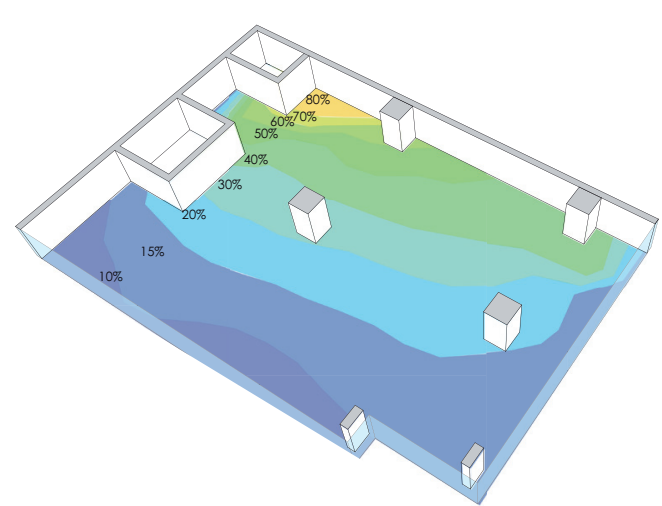

FIG.10 >

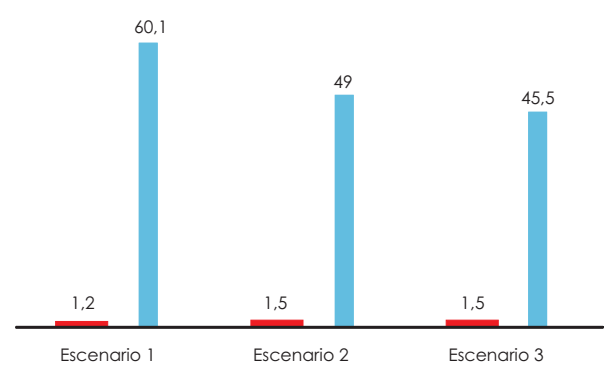

Calefacción $\mathrm{kWh} / \mathrm{m}^{2}$ año

Refrigeración $\mathrm{kWh} / \mathrm{m}^{2}$ año

FIG.08 > 
cina ocurren en el período de refrigeración (FIG.18). Además se observó sobrecalentamiento durante el verano, lo que obliga a ocupar estos sistemas de refrigeración. Sin embargo, en un día despejado de invierno este fenómeno tuvo menor intensidad que en ECM01, que es completamente vidriado y se encuentra en el mismo clima (FIGS. 7 y 17).

En ECM02 se observó que la demanda de calefacción fue mayor que la del edificio completamente transparente (ECM01) (FIGS.8 y 18). Para enfrentar esto, como primera medida, se mejoraró el estándar de las ventanas con DVH de tipo selectivo. Al hacerlo se observó que la demanda de energía de calefacción disminuyó solo levemente (FIG.18), lo que indicó la necesidad de optimizar también el muro de hormigón. Luego con la incorporación de aislante térmico de $50 \mathrm{~mm}$ en el muro, su transmitancia disminuyó significativamente, lo que permitió que la demanda de energía de calefacción descendiera a niveles muy bajos (FIG.19).

Respecto de la demanda de energía de refrigeración, las mejoras aplicadas para disminuir la demanda de calefacción no generaron un efecto significativo. Se desprende de aquí la necesidad de incrementar la ventilación nocturna.

En relación con el confort lumínico, aunque ECM02 tiene menor superficie vidriada, siguió mostrando un alto riesgo de deslumbramiento; sin embargo, se pudo establecer que el uso de protección solar exterior reduce significativamente. El índice UDI para rango entre 100 y 2.000 lux es extremadamente bajo cuando no se tiene protección solar (FIG.20), y con ello predomina la existencia de deslumbramiento porque la iluminancia estaría mayoritariamente sobre 2.000 lux.

VENTILACIÓN NOCTURNA / Con esta estrategia, la masa del edificio se enfría durante la noche para que al día siguiente dicha masa fría absorba calor del ambiente interno, lo que permite disminuir su temperatura. Se obtiene así una curva de temperatura amortiguada, con máximos retardados respecto de la temperatura externa (FIG.22). Cabe señalar que un muro de hormigón u otro material de alta densidad, cuya aislación térmica se instala por el interior, no ofrece inercia térmica al edificio pues su masa se expone hacia el exterior.

Se aplicó ventilación nocturna al caso ECM01, en su versión con protección solar externa. Esto permitió disminuir la demanda de refrigeración en un 7,1\%, aunque siguió siendo superior a la de ECM02, en su versión original. Por lo tanto este ejercicio no fue suficientemente efectivo por la escasa inercia térmica del edificio.

También se aplicó ventilación nocturna al caso ECM02. Se consideraron todos los mejoramientos aplicados, lo que disminuyó la demanda de refrigeración hasta límites significativamente más bajos que los alcanzados en ECM01 (FIGS.8 y 18). Esta caída fue de alrededor de un 37,5\% respecto de la situación original -escenario 1-. Si bien es probable que en un edificio de fachada mixta en clima mediterráneo la demanda de refrigeración llegue a límites inferiores, se observa que el conjunto de medidas aplicadas al edificio ECM02 permiten mejorar su comportamiento térmico, en todo el año, de manera muy significativa.

CONSIDERACIONES FINALES / El que la demanda de energía de calefacción haya sido -en los tres edificios-inferior a las respectivas demandas de enfriamiento, muestra que en edificios de oficina, con uso diurno intensivo, los problemas térmicos -y también lumínicos- son más críticos en verano y en ciertos meses de las estaciones intermedias. Por ello es que deben utilizarse estrategias de diseño tales como: vidrios selectivos, doble vidriado hermético (DVH), protección solar y ventilación nocturna.

A continuación, - en el texto final de esta edición-, se desarrolla un análisis de sensibilidad que permite conocer con mayor precisión el efecto combinado de estas y otras estrategias de diseño destinadas a enfrentar los problemas aquí detectados. +
FIG.11 A 14 ECC01. Edificio en clima costero 1. Fuente: elaboración propia

Especificaciones técnicas

Muro cortina: vidrio simple tipo reflectivo. Transmitancia solar (TS) $=0,47$.

Factor de transmisión de luz $(\mathrm{TL})=0,52$. Transmitancia térmica $(\mathrm{U})=5,8 \mathrm{~W} / \mathrm{m} 2{ }^{\circ} \mathrm{C}$.

Piso sobre subterráneo: hormigón armado (HA) $200 \mathrm{~mm}$.

Transmitancia térmica $(\mathrm{U})=2,2 \mathrm{~W} / \mathrm{m} 2^{\circ} \mathrm{C}$.

Cubierta: hormigón armado (HA) de $150 \mathrm{~mm}$. Transmitancia térmica $(\mathrm{U})=4,3 \mathrm{~W} / \mathrm{m} 2{ }^{\circ} \mathrm{C}$.

FIG.11 Esquema y planta.

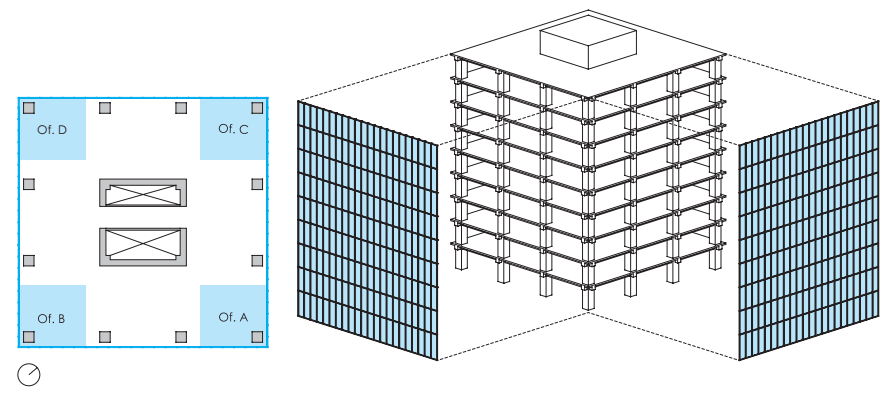

FIG.11 >
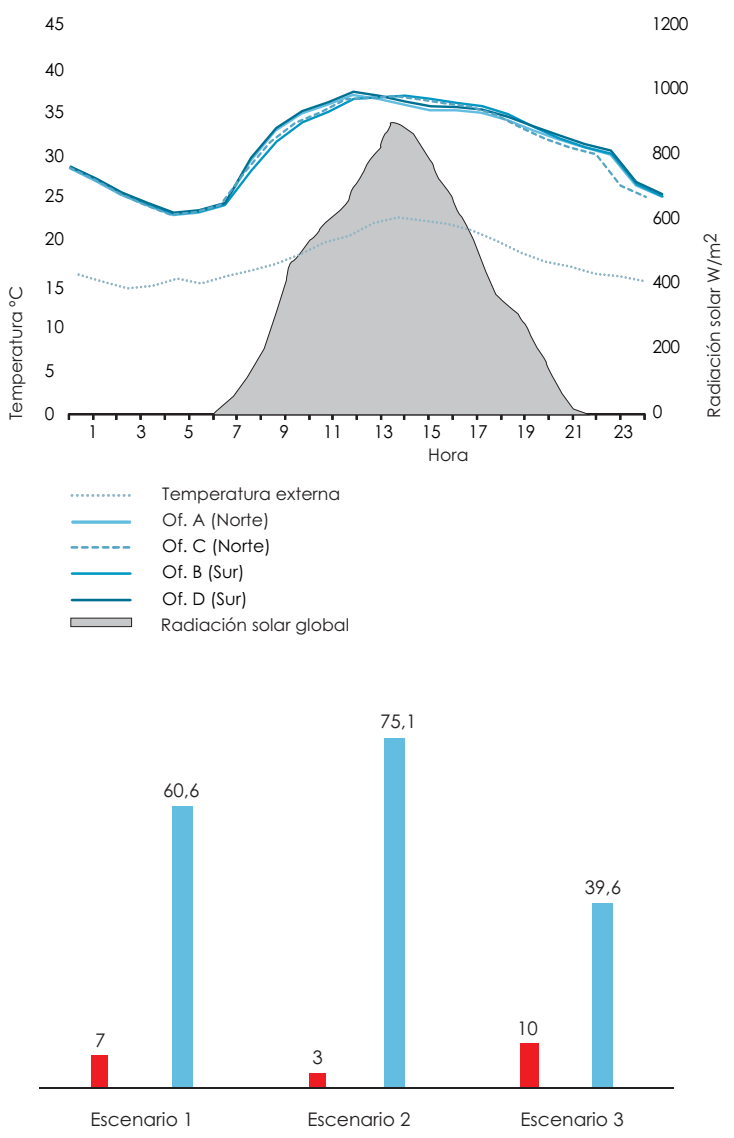

Calefacción $\mathrm{kWh} / \mathrm{m}^{2}$ año - Refrigeración $\mathrm{kWh} / \mathrm{m}^{2}$ año

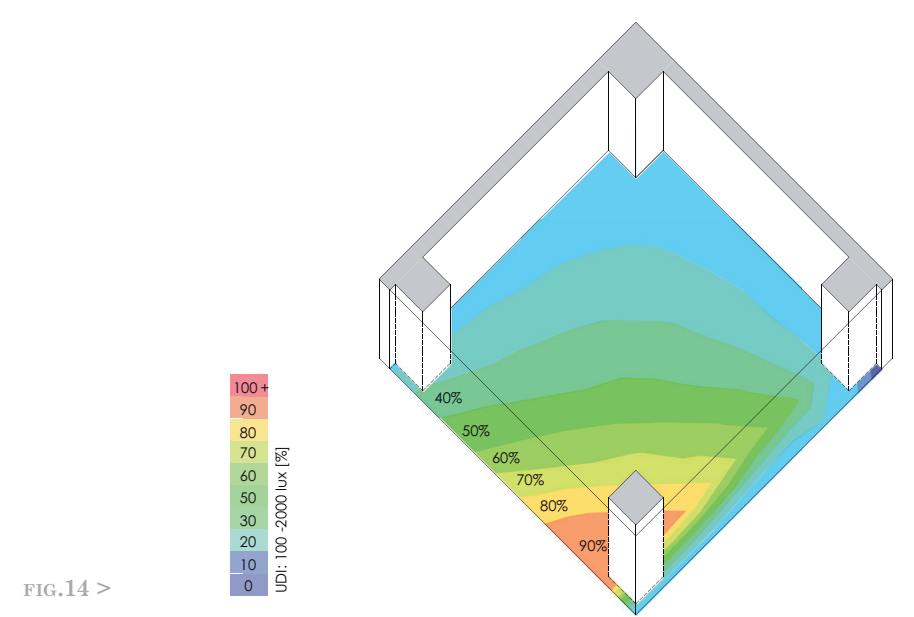


FIG.12 Variación de temperatura interior en recintos Este (oficinas A y B) y Oeste (oficinas C y D) en piso 5, en verano FIG.13 Demandas de energía de acondicionamiento térmico según diferentes escenarios.

Escenario 1: proyecto original.

Escenario 2: proyecto original con DVH reflectivo.

Escenario 3: proyecto original con protección solar: alero Norte y celosías Este y Oeste.

FIG.14 Iluminancia Natural Útil (UDI) para rango entre 100 y 2.000 lux en recinto interior del edificio. Muro cortina con vidrio simple reflectivo. Líneas de contorno de UDI: 100 a 2.000 lux cada $10 \%$

FIG.15 A 21 ECM02. Edificio en clima mediterráneo 2. Fuente: elaboración propia

\section{Especificaciones técnicas}

Ventanas: vidriado simple claro. Transmitancia solar (TS)= 0,87 y factor de transmisión de luz (TL) = 0,9. Transmitan cia térmica $(\mathrm{U})=5,8 \mathrm{~W} / \mathrm{m} 2{ }^{\circ} \mathrm{C}$.

Muro envolvente: hormigón armado (HA) $150 \mathrm{~mm}$. Transmitancia térmica $(\mathrm{U})=3,8 \mathrm{~W} / \mathrm{m} 2{ }^{\circ} \mathrm{C}$.

Piso sobre subterráneo: hormigón armado (HA) $150 \mathrm{~mm}$ con baldosa cerámica. Transmitancia térmica $(\mathrm{U})=2,31 \mathrm{~W} / \mathrm{m} 2{ }^{\circ} \mathrm{C}$ Cubierta: hormigón armado (HA) $150 \mathrm{~mm}$ y EPS $(10 \mathrm{~kg} / \mathrm{m} 3) 60$ $\mathrm{mm}$ de espesor. Transmitancia térmica $(\mathrm{U})=0,61 \mathrm{~W} / \mathrm{m} 2{ }^{\circ} \mathrm{C}$.
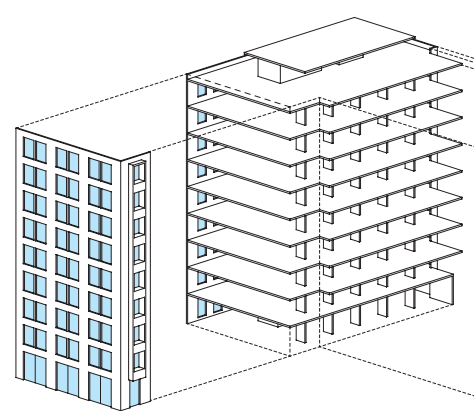

FIG.15 >
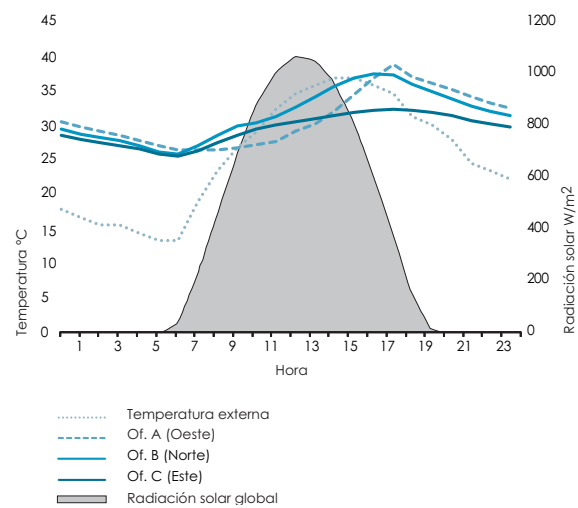

FIG.16 >

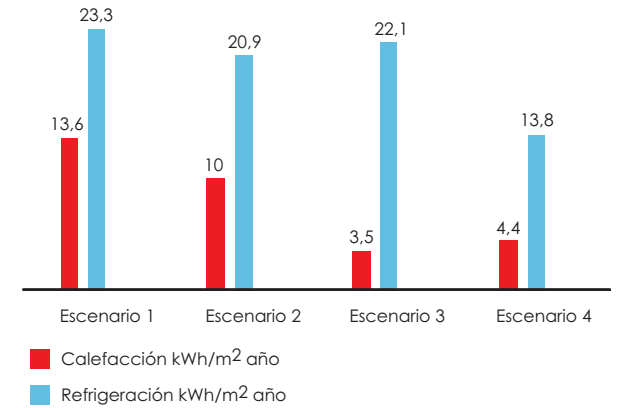

FIG. $18>$
FIG.16 Variacion de temperatura interior e.n recintos Oeste (of. A), Norte (of. B) y Este (of. C) en piso 5, en verano.

FIG.17 Variación de temperatura interior en recintos Oeste (of. A), Norte (of. B) y Este (of. C) en piso 5, en invierno.

FIG.18 Demandas de energía de acondicionamiento térmico según diferentes escenarios.

Escenario 1: proyecto original.

Escenario 2: proyecto original con DVH selectivo. Transmitancia solar $(\mathrm{TS})=0,4$ factor de transmisión de luz $(\mathrm{TL})=$ 0,5 . Transmitancia térmica $(\mathrm{U})=2,8 \mathrm{~W} / \mathrm{m} 2{ }^{\circ} \mathrm{C}$.

Escenario 3: escenario 2 con aislación térmica exterior de 50 mm (muro) y protección solar en todas las ventanas: vertical en fachadas poniente y oriente y alero en ventanas norte. Escenario 4: escenario 3 con ventilación nocturna -renova ción de aire de 8 volúmenes por hora, entre 22:00 y 07:00 hr del día siguiente-, entre octubre y marzo.

FIG.19 Sistema constructivo escenarios 3 y 4 .

FIG.20 Iluminancia Natural Útil (UDI) para rango entre 100 y 2.000 lux en recinto D del edificio. Sin protección solar exterior.

FIG.21 Iluminancia Natural Útil (UDI) para rango entre 100 y 2.000 lux en recinto D del edificio. Con protección solar exterior

FIG.22 Curvas de temperatura interior con y sin ventilación nocturna. Fuente: elaboración propia

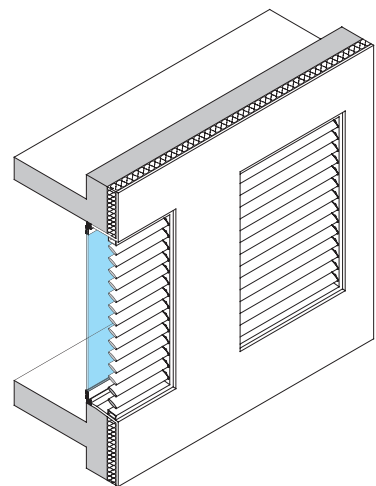

FIG.19 >

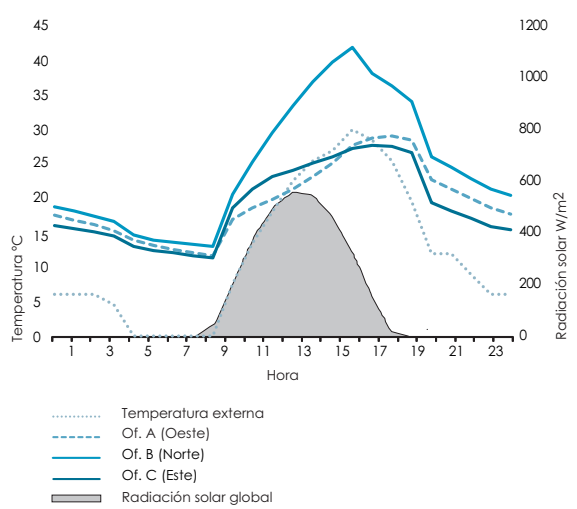

FIG.17 >

Bibliografía sugerida

Bresesh, H., Bossaer, A. y A. Janssens. "Passive cooling in a low-energy office buinding. Solar Energy, $\mathrm{N}^{\circ}$ 79, Polymeric Materials for Solar Energy Applications. Elsevier, Ámsterdam, diciembre de 2005. Bustamante, Waldo y Yoselin Rozas. Guía de diseño para la eficiencia energética en la vivienda social. Ministerio de Vivienda y Urbanismo, Santiago, 2009 / Salomon, Thierry. Fraîcheur Sans Clim': Le Guide Des Alternatives Ecologiques. Terre Vivante Editions, Mens, 2004 